\title{
THE ECONOMIC ASPECTS OF THE METROPOLITAN REGION*
}

\section{Britton Harris $\uparrow$}

In the very nature of the title of this article, there are perhaps three major problems which ought to be clarified. First, perhaps, is the importance of the region, especially the "natural" region. This receives attention elsewhere in this symposium and is relatively the least important point in my discussion although it will deserve some attention. The second is the distinction between metropolitanism and other aspects of regionalism which are of importance especially from the economic point of view. This also requires some elaboration. Finally, there is the main problem to which this article is addressed, that is, the economic aspects of the metropolitan region itself. These economic aspects will be substantially clarified in the process of definition.

\section{Delineation of the "Region"}

The existence or nonexistence of regions and the criteria by which regions may be distinguished from each other have been endlessly debated. Even on a featureless plain any pattern of settlement would tend to become "regionalized" because of the difficulties of communication. A region would be at least an area each part of which is further from any other regional center than from its own center. There is, however, more to regionalism than pure separation in space. At the risk of oversimplification, I would tend to adopt the working hypothesis that regions exist as the result of natural forces, and that these forces tend to make the boundaries of regions-as marked off by different criteriacoincide closely with each other. On the other hand, the strong social and political forces which operate to produce uniformity within the United States tend to break down regional cultural differences, and still other forces (especially migration) tend to break down regional economic differences. While regions will therefore continue to exist, regional distinctions will become less important.

* This article being in the nature of a thoughtful but impressionistic review of the title problem, it has seemed impractical to select a few out of the growing body of references on the topic. The author is nonetheless indebted to the work of many in this field, and especially to the stimulating contacts provided with his colleagues and others through his work at the Institute for Urban Studies.

$f$ Project Director, Institute for Urban Studies, University of Pennsylvania; Formerly Director of Economic Research, Economic Development Administration of Puerto Rico. B.A., 1935, Wesleyan University; M.A., 1951, University of Chicago. 
The natural configuration of land, wind and climate tends to mark off a great land mass into roughly quadrilateral areas. Crossing any land mass on an east-west line, we encounter different zones of rainfall and soils, and different degrees of accessibility to the ocean and to other areas, all of which are conditioned by the general wind pattern and the succession of land forms which intercept these winds, control precipitation, provide the material for soil formation and carry resources such as minerals beneath the surface or hydroelectric sites on the surface. Movement in a north-south direction carries us across the zones of differing average temperatures which affect soils, rainfall and crop-carrying capacities. Except at rugged mountain tops none of these transitions is abrupt-yet the differentiation is sufficiently marked to enforce very clear patterns of regional development within "homogeneous" zones marked out on all four sides. This differentiation has occurred over the long period when the exploitation of natural resources, including agriculture, was the dominant activity of man. Thus, it is no accident that slavery did not extend north of the Mason-Dixon line, and, hence, that the culture of the South differs from that of the North. Neither is it an accident that agriculture in New Jersey differs from that in Illinois, Wyoming and Oregon, and that there are associated cultural and social differences arising in part out of economic conditions and in part out of the history of settlement. In areas with a longer history of settlement than the United States, it is not unusual to find this differentiation extending to such advanced cultural manifestations as language, a differentiation made possible by barriers to easy communication.

Thus, differentiation within any large area leads to the demarcation of regions-however broadly or narrowly defined. These regions have the common characteristic of some degree of physical and climatic uniformity, leading directly or indirectly to differences in economic activity and culture. These differences are reflected not only in the extractive activities based on land, but in the character and base of the cities which serve the region. Hence to some considerable extent, cities may be viewed as a specialized form of settlement within a larger natural region. The growth in size and importance of the larger cities, however, has cast some doubt on this framework, and has led to special consideration for the "metropolitan region."

The metropolitan region as a concept and a method of classifying areas presents more inherent difficulties than does the "natural" region. The presence of these difficulties does not negate the concept; they arise in fact out of the very circumstances which bring about the existence of such regions. 
A metropolis is a large city (though it is more than that), and its history is the history of cities. Without entering into involved discussion of some of the more subtle problems in this connection, it is clear that the growth of the city rests upon increased productivity and the division of labor. Without higher agricultural productivity, city dwellers could not be assembled and fed, but would remain tied to the land. Once cities became possible, they gradually began to play the role of trade marts, workshops and centers of culture-governmental-ecclesiastical and lay. With the explosion of technology and political revolution of the seventeenth and eighteenth centuries, the city came into its own as the growth center of a new and powerful social organization. Such cities were based on new functions, of which manufacture was the most important, and lived not only on political domination of the surrounding countryside, but on a crescent exchange of commodities and services within whole nations and empires. At the same time they exploded physically outside the walled bounds of the medieval burg or fortress, initiating an expansion which is not yet at an end-even in cases where it embraces ten million or more people and covers several thousands of square miles.

Because of the distinctive characteristics of the modern metropolis, it has become fashionable to refer to the "metropolitan region" as a unit of analysis. Such a region, however difficult it may be to define its boundaries, is clearly distinct from its "hinterland" in its density, in its urbanity, and in the interdependence of its parts. It is "nucleated" around an important central point or core-and this characteristic may help to set it off from neighboring metropolitan regions. These attributes are so important as to override the very real difficulties of this unit of analysis.

In most uses of this concept, we find that the metropolitan regions do not exhaustively divide a national territory. There usually remain interstitial areas where human occupancy is based on the natural characteristics of the land as a directly productive element in economic activity. One can, of course, delimit zones of influence of major cities (somehow defined), so that the whole area is divided among them. On the basis of wholesale trade, banking, utilities, newspaper circulation or radio and TV coverage, this gives a number of similar divisions of the whole area into metropolitan service areas. Yet this division is fundamentally unsatisfactory-one continues to feel that the relation of North and South Dakota to Minneapolis is more tenuous than that of New Brunswick to New York City or of Oakland to San Francisco.

This fact is, of course, recognized by the Census Bureau definition of the Standard Metropolitan Area, which includes contiguous urban- 
ized counties, economically related to a central city of 50,000 or more inhabitants. The economic relation is usually measured by the level of commuting to work, either into or out of the central city. Such an area coincides closely with those measured by other criteria, such as department store retail trade, and it satisfies the intuitive search for some measure of integration within the metropolitan area. Unfortunately, the small size of the minimum central city, which elevates Peoria, Illinois, and Wilmington, Delaware, to the level of the metropolis, makes the definition belie the name and leads to a needless confusion between urbanism and metropolitanism. Yet because of its statistical convenience and its obvious pertinence to modern problems, the Standard Metropolitan Area is here to stay. We will focus our attention on the larger and more populous of these areas, which have some claim to being more than just cities.

The inability of this definition-of a metropolitan region which is internally highly organized-to divide up completely the whole nation, leads to some frustrations. Yet the interstitial areas can be assigned to "their" metropolitan centers only by main force. Middle Western wheat is milled in Buffalo as well as in nearer centers; fruitgrowing and truck-farming in California, Colorado and Texas are "tributary" to New York as well as to Los Angeles, Denver and Dallas. In short, the hinterland of one city is a hinterland for many cities, and a metropolis which performs one function for a surrounding area may perform others for areas thousands of miles away. The urban and metropolitan centers of the United States, which now contain close to two-thirds of our population, perform a manifold variety of functions, some of which reach only around the corner, some of which serve a conventional hinterland and stop at the borders of the "next" hinterland, and some of which reach around the world.

\section{Movement, Change and Growth Within the Region}

It is convenient, both between metropolitan centers and between neighborhoods within the metropolitan centers, to think of a hierarchy of functions, each more specialized than the one below it. In most functions the hierarchy exists as to actual content-this is so in government or trade, for example. In others the ranks are not functionally related, but differ purely in degree of specialization. In this view, small cities have mainly the functions which small torens cannot perform economically-and they have them in common with all other small cities. Large regional centers have certain mutually similar functions which no smaller city can discharge; and at the same time 
perform small city functions on a large scale. A few cities like New York and Washington have, in addition to all these previous functions, special roles which are unique. Thus the division of labor and specialization also leads to a hierarchy of metropolitan centers, of which the larger and more complex have a greater multiplicity of functions in the economy as a whole.

Similar observations apply to the differentiated parts of a metropolis-where the greatest multiplicity and complexity of function is confined to the central business district, which also contains functions whose discharge is repeated in satellite cities, in large shopping centers, and in neighborhood centers.

From an economic point of view, the obverse of specialization is movement. When functions are divided between people, between neighborhoods, between cities, between regions, and between the metropolitan areas and the rural areas as a whole-then each can subsist only by an exchange of products, of goods and services, with the others. Not only the unity, but also the productivity, of the nation depends to a very great degree upon this mobility, upon a high volume of transportation and communication. Within each metropolitan region, this mobility reaches a peak. Specialization is fostered by the ability of any workplace, any producer, to reach out and assemble his needs from a variety of personal skills, services or stocks of materials. At the same time, this assemblage of firms and people is a market for his (possibly specialized) products.

As the author once heard Luther Gulick informally remark, the city and the metropolis owe their unique position to the fact that they facilitate circulation - the circulation of goods, the circulation of people and the circulation of ideas. The businessman recognizes this unique function when he considers the location of his establishments, and the more dependent he is either upon the markets of the metropolis or upon the availability of goods, services and labor, the closer he will be to a metropolitan center. It is no accident that the large cities of this country have the widest diversity of economic activities, and tend to have smaller average sizes of establishments in manufacturingindicating their role as "breeders" of new economic activity and innovation. The individual in search not only of economic opportunity, but of diversity of stimulus and greater self-realization, is also drawn to the larger cities. Thus the continued march of specialization of activity (even with increased uniformity of consumption and culture), tends to make large cities grow more so. Their growth is fed by the declining importance of extractive industries and by the failure of the 
smaller towns and cities to exercise similar attractions and to grow in any similar way. Nothing succeeds like success.

Reflection will indicate that while the movement of goods is the essential feature of the regional differentiation of activity, within the metropolitan area the movement of people is the essential and primary factor leading to effective economic activity and growth. The people of the area must be fed and clothed and housed, and productive establishments must be supplied with materials; these lead to important but subsidiary types of goods movements which re-enforce the need for concentration. Without the specialization and scale of production which requires such a diversity of skills and such a number of employees in many establishments, the reason for existence of the metropolitan region would lose its force. The intuitive validity of a definition based on labor market areas and commuting is thus confirmed. The metropolitan region is not simply an area in which circulation reaches a higher density; it is an area in which a certain type of circulation, the journey to work, is of paramount importance and binds the entire region together.

The foregoing distinction is to some extent incomplete; it tends to set off metropolitan regions from "natural" regions, but it includes too many smaller cities. It clearly denies an independent role to the satellite cities of a metropolis, but it does not exclude from the category of metropolis isolated cities of equal or smaller population, which have no organic tie with larger centers through the journey to work. These must be ruled out simply on the basis of inadequate size, complexity and multiplicity of function. Such a rule will be arbitrary as between some two or more cities which are very similar, but further analysis would show that, among cities, differences in size beget differences in quality which demand new categories. At some point around a population of 500,000 , the city of today becomes a metropolis and has "metropolitan" problems.

Economically, these problems are associated with growth, in relation to the functions which sustain that growth. As metropolitan areas have continued to grow in size and complexity, they have historically been confronted with crises of circulation. These have been solved by innovations in transportation and metropolitan organization which permitted new growth and created new problems of their own. At various stages, such growth has spread across city political boundaries, and the resulting strains have been sufficiently acute to appear to be "the" metropolitan problem, or at least its most basic cause. Such political problems are most important, and are wisely the focus for much of this symposium, but they would not be problems 
were it not for even more fundamental aspects of function and disfunction in the metropolitan structure. These underlying difficulties arise out of size and growth, and will always be present in a dynamic metropolitan area, regardless of its governmental organization. Since meeting these difficulties usually requires government action, the fragmentation of jurisdictions in most great cities becomes the form in which all difficulties appear. It is no more than a part of their substance.

At this point we may make a brief reference to one supposed problem which has by now become almost but not quite academic. We have posed problems of growth, which will shortly be discussed in more detail. We will mention in brief the "declining" metropolitan area, or the region which is not fully participating in national growth. The problems of such areas will not be as acute as was once anticipated, since we can no longer expect the national population to approach a stationary level, nor the economy as a whole to- stagnate. There are, therefore, more recovery opportunities for an area like New England whose old regional specializations became obsolete or non-competitive. Such areas still have a lower than average growth potential, and thus less than average resources with which to meet their common metropolitan problems. They become special and more acute cases of the general malaise which we are examining.

Growth and change inflict their penalties on all areas, however. Our large cities are all to some degree crowded, congested and unpleasant. They suffer social and economic disabilities which are to some extent a reflection of their physical organization and the obsolescence of their structures. All of this is quite distinct from those problems of social, political and economic organization which are of national import and which are to be observed in the cities because people live in cities. Despite these shortcomings, it may confidently be predicted that within the next twenty-five years, the population of nearly every large metropolitan area will increase by at least half, and within fifty years by as much as one hundred per cent. With this will go an increasing "suburbanization" or decrease in density. As a result, the size and extent of metropolitan areas will increase enormously, and with them the difficulties of circulation and of the supply of services. Together with growing obsolescence, these are the basic problems of function which already beset our metropolitan areas, and which are bound to become more acute. Economically, the problems have two sides. On the one hand, a collapse or impairment of metropolitan function will strike at the root of national economic growth and effectiveness. On the other, metropolitan areas as such generate but do not control the resources which would be needed to resolve these 
problems. The present and impending crisis in metropolitanism is therefore of national significance.

\section{A Closer Examination of Regional Problems}

Let us examine a little more closely the actual texture of the difficulties. The age of the automobile has revolutionized the internal circulation pattern of urban areas, just as did other changes before it. Population is rapidly being redistributed within these areas, which are receiving as a whole almost all of the national growth of population. The central cities are standing still, and the suburbs are growing in night-time population at a rate four times the national rate of growth. Although it has been noted (and sometimes with alarm) that manufacturing and trade are also suburbanizing, their rate of movement out of the central city is far less precipitate. It therefore follows that the volume of movement within these areas, especially to and from work, is growing at as least as rapid a rate as population. Within the central city, the stock of housing and other improvements continues to age, and present public resources and powers are inadequate to check the spread of blight. This danger is exacerbated by the differential movement of population to the suburbs by income, occupation, social status and race, which imposes upon the central city the burden of rising costs in social services. Finally, all sectors of the population have rising incomes and rising needs. The demands upon governments for education, recreation and "facilitating" services, which make it easier to spend more income, are rapidly increasing.

Thus each part of the metropolitan area has its distinctive problems. The suburbs must accommodate a rapidly increasing adult population, and their children present and future. They must provide roads, schools, open space, water, sewerage and a multitude of new services. The central city must accommodate a relatively unskilled in-migrant population, cope with growing social problems and tackle an increasing backlog of redevelopment and rehabilitation. It must provide central services, especially those connected with communication and circulation, for a growing business community and a growing total area. The whole metropolis faces needs for circulation, sanitation and other fundamental services to which no single portion of the area can contribute adequate support, even with existing state and federal assistance.

If local taxes can be taken as an indication of the impact of these problems, insofar as they are being met, it is of utmost importance to 
note that suburban areas are rapidly approaching the central city in their level of taxation. It can no longer be said that the impact of these needs is completely inequitable as between the city and its suburban ring, except insofar as the central city faces greater future problems. At the same time the present high and rising level of all local taxes, together with a growing backlog of urgent work facing local governments, indicates that the problem of metropolitan services is beginning to exceed the grasp of local action.

It may be argued that metropolitan functions benefit both the business establishments and the residents of the area and that the needed increase of services should rightly be paid out of local resources, either through conventional methods of financing or through a sharp increase in user taxes. This contention is not altogether in the realm of economic analysis, but raises sharp problems of political and social policy, which are discussed elsewhere in this symposium. Economically speaking, however, this does raise the question of competition between metropolitan areas and between jurisdictions within metropolitan areas, especially in the effort to secure the advantages of increased industrial location. Such competition is desirable when it is carried out through an increase of amenity and efficiency by public action. Its usefulness in the national setting may be questioned when it results in a cut-back in services or in an increase in taxes which are essentially regressive. One of the most potent arguments for the unification of local jurisdictions or for the increase of national and state assistance to metropolitan areas lies in the fact that it would make such competition unnecessary. This would be a significant by-product of whatever thorough-going measures are finally determined upon to meet the tremendous needs of metropolitan reorganization.

Settlement of these problems in the political sphere will require facing certain economic facts. Unified action within the metropolitan region will be achieved only if both the central city and the suburbs become conscious of the fact that they function as a unified economic entity, in which the most important problems remorselessly pursue the citizen across political boundaries. Neither the city nor the suburbs can afford to neglect or overlook the problems of the other. On the state and national level, at least two bits of political folklore are due for some re-evaluation. One is our unwillingness to treat any but farm problems as special area problems affecting the national economic and social health. Urban and metropolitan problems are not approached together as a single set of problems affecting metropolitan areas, but by the back door of piecemeal legislation on specific problems such as slums and housing-which happen to arise mainly in cities. As 
the magnitude of the resources needed to solve metropolitan problems rapidly increases we are also likely to see less emphasis upon complete local independence from larger units of government, and some tempering of the insistency upon economy in government-at least where such economy would deprive large and vocal groups of much-needed services. We are, finally, likely to discover through efforts to apply artificial measures for decentralization and dispersion in the interest of national defense, that the metropolitan community is an organism which cannot easily be tampered with or turned aside from its normal path of development.

If this last supposition is correct, and there is every indication that it is, the problems which we have outlined take their source deep in our economic and social organization. The growth of metropolitan areas assumes the aspect of a natural force. This force will bring increasing millions of people into contact with metropolitan problems. Their education in, and understanding of, these problems will come as a result of sometimes bitter experience. At increasing levels of income they will tolerate less inconvenience, congestion, dirt and squalor. They will tolerate less distraction from their leisure time and facility of movement. After many false trials they will insist upon rational solutions to these problems and will countenance the very large expenditures which will be required to put these solutions into effect. This will be so because, after the overwhelming problems of national defense and economic security, the problems of metropolitan development have the greatest implications for continued economic growth, and the strongest impact on the daily lives of a growing proportion of our population. 